\title{
Correction to: Decentralized Platform Ecosystems for Data-Sharing and Digital Trust in Industrial Environments
}

\author{
Kilian Schmück, Monika Sturm, and Oliver Gassmann
}

\section{Correction to:}

Chapter 7 in: O. Gassmann, F. Ferrandina (eds.), Connected Business, https://doi.org/10.1007/978-3-030-76897-3_7

The original version of the chapter was inadvertently published with an error. The chapter title has now been corrected to "Decentralized Platform Ecosystems for Data-Sharing and Digital Trust in Industrial Environments". 